\title{
FINGER KNUCKLE PRINT BASED VERIFICATION USING MINIMUM AVERAGE CORRELATION ENERGY FILTER
}

\author{
Gaurav Verma \\ Indian Institute of Technology Delhi \\ Department of Physics, Indian Institute of Technology Delhi, Hauz \\ Khas, New Delhi-110016, India \\ gaurav.sgs85@gmail.com \\ Aloka Sinha \\ Indian Institute of Technology Delhi \\ Department of Physics, Indian Institute of Technology Delhi, Hauz \\ Khas, New Delhi-110016, India \\ aloka@physics.iitd.ac.in
}

\begin{abstract}
Biometric-based technologies are being widely used for personal authentication and identification in access control and e-commerce applications. Finger knuckle print, a new hand based biometric trait has recently been utilized in verification and identification based systems. A finger knuckle print possesses a unique and highly distinctive pattern. In this paper, the high discrimination capability of correlation filters were employed for a finger knuckle print based recognition system. The correlation filters have important characteristics like shift invariance and distortion tolerance. A minimum average correlation energy correlation filter was designed for finger knuckle print verification. The performance of the designed filter was evaluated by calculating the peak to side lobe ratio, the false acceptance ratio, the false rejection ratio and the equal error rate. The computational experiments were done on a Matlab platform on the Poly $\mathrm{U}$ finger knuckle print database.
\end{abstract}

Keywords: Authentication, Correlation Filter, Minimum Average Correlation Energy Filter, Finger Knuckle Print, Region of Interest 


\section{INTRODUCTION}

Automated methods based on biometrics are used to authenticate and identify users. These are based on human characteristics and can be classified into two categories: (i) physiological characteristics, such as inherent physical traits such as a fingerprint, face, palm print, iris, retina; and (ii) behavioural characteristics, which are learned or acquired such as signature, typing rhythm, gait, voice and signatures ${ }^{1}$. Biometric-based methods possess advantages over traditional methods since there is no need to remember something or fear theft or hacking of a password, pin and token. Biometrics are also always present with the associated person.

Among the various kind of biometrics traits, the finger knuckle print is an emerging biometric trait. A unique and highly distinctive pattern is obtained when the finger knuckle is bent and this is useful for recognition. As a type of hand-based biometric, a finger knuckle print has ease of acceptance and is highly convenient. It has a very high potential for recognition of a person. It offers several advantages over various kinds of biometric traits such as face, fingerprint, palm print and iris recognition ${ }^{2}$. The disadvantages associated with face recognition are that the system performance varies with expression, wrinkles, beard, and eyeglasses over time. In the case of a fingerprint, there is an inherent stigma associated with a potential criminal investigation. Palms are large in size and include rich features such as creases, palm lines, ridges, and minutiae. It is hard to copy the complete palm print. An iris-based system offers distinctive information for personal authentication and requires a higher degree of user cooperation.

Correlation filters have been successfully used for personal authentication systems based on different biometric traits. Savvides et al. proposed a correlation filter for face verification using a minimum average correlation energy (MACE) filter ${ }^{3}$. Venkataramani et al. investigated correlation filters for fingerprint verification ${ }^{4}$. Kumar et al. reported the use of correlation filters for iris verification ${ }^{5}$. Finger knuckle print has recently been employed in biometric-based recognition systems.

The utilization of a finger knuckle print for verification was first proposed by Woodard and Flynn ${ }^{6}$, however, practical implementation was not possible. Kumar et al. ${ }^{7}$ proposed an approach for personal authentication using a finger knuckle surface. Zhang et al. proposed a band-limited phase-only correlation filter for finger knuckle print authentication ${ }^{8,9}$.

This paper proposes the use of a minimum average correlation energy filter for finger knuckle print verification. In order to design a correlation filter for finger knuckle print verification, the region of interest of the finger knuckle print has to be extracted. 
The rest of the paper is organized as follows. Section 2 describes the region of interest extraction process of a finger knuckle print. Section 3 discusses the process of designing the minimum average energy filter and the process of recognition using FKP verification. Section 4 investigates the performance of the filter. Section 5 reports the results and discussion. Finally, the conclusions are presented in Section 6.

\section{THE REGION OF INTEREST EXTRACTION PROCESS}

The finger knuckle print region of interest (ROI) extraction process has been proposed by Zhang et al. ${ }^{9}$. In order to extract the ROI of a FKP, a rectangular coordinate system is configured for determining the $\mathrm{X}$-axis and Y-axis. First, a Gaussian filter is used to smoothen the original FKP image. After that a Canny edge detector is applied to the smoothened FKP image which produces sharp discontinuity and line features in the image. An approximate cropped sub image of FKP is obtained based on the left and the right fixed boundaries values and the top and bottom boundary values of the FKP images. This cropped sub image is used further to determine the Y-axis of the coordinate system. The Canny edge detector is again applied on this cropped sub image. This produces the corresponding edge map of the FKP image $\left(\mathrm{I}_{\mathrm{CD}}\right)$. The convex direction coding is used to code the pixels on the convex leftward and rightward curve as ' 1 ', and the other pixels not on any curve as ' 0 ' on an edge map of the FKP image. This observation is used to decide the $\mathrm{Y}$-axis coordinates by calculating the minimum convex magnitude as given in Eq. (1).

$$
\operatorname{conMag}(\mathrm{x})=\operatorname{abs}\left(\sum_{\mathrm{w}} \mathrm{I}_{\mathrm{CD}}\right)
$$

A window ' $w$ ' is chosen of size $(b \times h)$ where, $b$ is the width of window and $h$ is the height of image $\left(\mathrm{I}_{\mathrm{CD}}\right)$. The minimum magnitude value of the image is determined at the point $X=X_{0}$ and is given in Eq. (2) as:

$$
\mathrm{x}_{0}=\arg _{\mathrm{x}} \min (\operatorname{conMag}(\mathrm{x}))
$$

Finally, the rectangular coordinate is determined. In the present study, the original FKP image was cropped at a fixed size of $110 \times 221$ pixels at about the $\mathrm{X}$-axis and the Y-axis. The computation time for extraction of ROI for one FKP was found to be 0.570348 seconds on an Intel Core i5-2450M processor with $2.50 \mathrm{GHz}$ and $4 \mathrm{~GB}$ RAM. Figs. 1(a) and 1(b) show the original FKP image and the ROI extracted image of FKP, respectively. 


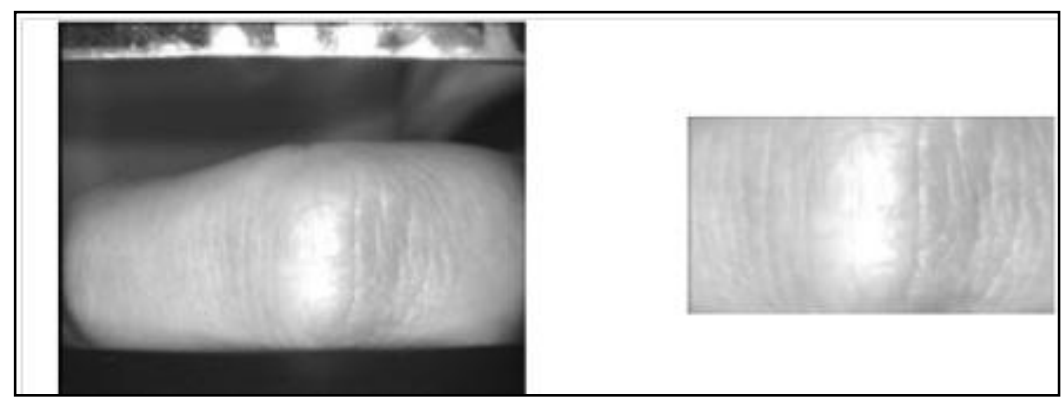

Figure 1. (a) Original FKP image (b) Extracted ROI of FKP image

\section{MINIMUM AVERAGE CORRELATION ENERGY FILTER}

Correlation filters are widely used in pattern recognition based applications like automatic target recognition and biometric-based recognition systems ${ }^{10}$. A basic space invariant correlation filter is known as the matched spatial filter (MSF) whose impulse response is the complex conjugate of the reference image ${ }^{11}$. The MSF is used to maximize the signal-to-noise ratio if a reference image is present in additive white noise. It works inadequately when there is distortion or scaling of the reference image. The synthetic discriminant function (SDF) filter uses a multiple training set of images of an object made from different orientations of the image. The shortcoming of SDF filters is that they produce a large side lobe and lack sharp correlation peaks. As a result, the SDF filter shows a low discrimination capability ${ }^{12}$. Kumar et al. presented a study of different correlation filtering techniques and the performance criteria for comparison among the various kind of correlation filter designs such as signal-to-noise ratio, peak sharpness, peak location, light efficiency, discriminability, and distortion invariance ${ }^{13}$. The problems and limitations of MSF and SDF filter are overcome by using the minimum average correlation energy filter. Mahalanobis et al. ${ }^{14}$ proposed the minimum average correlation energy filter (MACE). The MACE filter is used to minimize the average correlation plane energy and at the same time it satisfies the pre-specified peak value at the origin. This filter was found to be more effective for reducing noise and side lobes in the correlation plane. It also produces a sharp correlation peak at the position of the trained object in the correlation plane.

A set of finger knuckle print images are used to train the filter. This can be represented by column vector matrix $X_{i}(m)$ in space domain as given in Eq. (3).

$$
\mathrm{x}_{\mathrm{i}}(\mathrm{m})=\left[\mathrm{x}_{1}, \mathrm{x}_{2},-----\mathrm{x}_{\mathrm{i}}\right]
$$


where, $X_{i}$ is the ith finger knuckle print and the size of each of the finger knuckle print image is $d_{1} \times d_{2}$ pixels. The Fourier transform (2-D FFT) is performed on these training images ${ }^{15}$. The 2-D FFT arrays are then converted into a one-dimensional column vector (X) of size $\mathrm{d} \times \mathrm{N}$ by concatenating as given below:

$$
\mathrm{X}=\left[\mathrm{X}_{1}, \mathrm{X}_{2},----\mathrm{X}_{\mathrm{N}}\right]
$$

where, $d=d_{1} \times d_{2}$ are the total number of pixels in the image and $\mathrm{N}$ is the number of training images. The vector $\mathrm{h}(\mathrm{m})$ and $\mathrm{H}(\mathrm{u})$ are used to represent filters in the space and frequency domains, respectively. The correlation output $g_{i}(m)$ is calculated by correlating the training image sequence $X_{i}(m)$ with the filter $h(m)$ as given below:

$$
\mathrm{g}_{\mathrm{i}}(\mathrm{m})=\mathrm{x}_{\mathrm{i}}(\mathrm{m}) \square \mathrm{h}(\mathrm{m})
$$

The calculated correlation plane energy $\left(E_{i}\right)$ is given as:

$$
\begin{aligned}
\mathrm{E}_{\mathrm{i}} & =\sum_{\mathrm{m}=1}^{\mathrm{d}}\left|\mathrm{g}_{\mathrm{i}}(\mathrm{m})\right|^{2}=\left(\frac{1}{\mathrm{~d}}\right) \sum_{\mathrm{u}=1}^{\mathrm{d}}\left|\mathrm{G}_{\mathrm{i}}(\mathrm{u})\right|^{2} \\
& =\left(\frac{1}{\mathrm{~d}}\right) \sum_{\mathrm{u}=1}^{\mathrm{d}}|\mathrm{H}(\mathrm{u})|^{2}\left|\mathrm{G}_{\mathrm{i}}(\mathrm{u})\right|^{2}
\end{aligned}
$$

The correlation output function $G_{i}(u)$ is represented in the frequency domain and the Eq. (6) is simplified by using Parseval's energy theorem. This equation can be further represented in vector form as:

$$
\mathrm{E}_{\mathrm{i}}=\mathrm{H}^{+} \mathrm{X}_{\mathrm{i}} \mathrm{X}_{\mathrm{i}}^{*} \mathrm{H}=\mathrm{H}^{+} \mathrm{D}_{\mathrm{i}} \mathrm{H}
$$

where, the '+' symbol represents the complex conjugate transpose and $D_{i}$ is the diagonal matrix in which the diagonal elements are equal to the magnitude square of the elements of the training image set $X_{i}$. The sum of the entire diagonal matrix $\left(D_{i}\right)$ is represented by $D$.

The average correlation energy can be calculated as:

$$
\mathrm{E}_{\mathrm{ACE}}=\frac{1}{\mathrm{~N}} \sum_{\mathrm{i}=1}^{\mathrm{N}} \mathrm{E}_{\mathrm{i}}=\mathrm{H}^{+}\left[\frac{1}{\mathrm{~N}} \sum_{\mathrm{i}=1}^{\mathrm{N}} \mathrm{X}_{\mathrm{i}} \mathrm{X}^{*}\right] \mathrm{H}=\mathrm{H}^{+} \mathrm{D} \mathrm{H}
$$

The correlation peak values for each training images $X_{i}$ have been constrained by inserting the pre-specified column vector $C$ which consists of elements ' $C_{i}$ '. These values are set to $C_{i}=1$ for images of the true class 
and $\mathrm{C}_{\mathrm{i}}=0$ for a false class. Thus, $\mathrm{H}$ must fulfill the pre-specified peak constraint condition as given in Eq. (9).

$$
\mathrm{H}^{+} \mathrm{X}_{\mathrm{i}}=\mathrm{C}_{\mathrm{i}}
$$

For all images, this can be written as

$$
\mathrm{H}^{+} \mathrm{X}=\mathrm{C}
$$

Hence, the solution of the MACE filter is guided by minimizing the average correlation energy $\left(\mathrm{E}_{\mathrm{ACE}}\right)$ in Eq. (8) while satisfying the peak constraint in Eq. (10). The final optimal solution of the synthesized MACE filter is obtained in vector form as given below:

$$
H=D^{-1} X\left(X^{+} D^{-1} X\right)^{-1} C
$$

\subsection{Recognition Process of FKP}

In the present study, three finger knuckle print training images were utilized to design a single correlation filter for each person. The test image was then cross-correlated with the designed filter and the obtained correlation output was then used to decide whether it was a genuine image or an impostor image. The output of the designed correlation filter for the genuine case has a strong peak, but in the case of an imposter, multiple peaks of low peak values were obtained. The recognition process of the finger knuckle print verification using MACE filters is shown in Fig. 2.

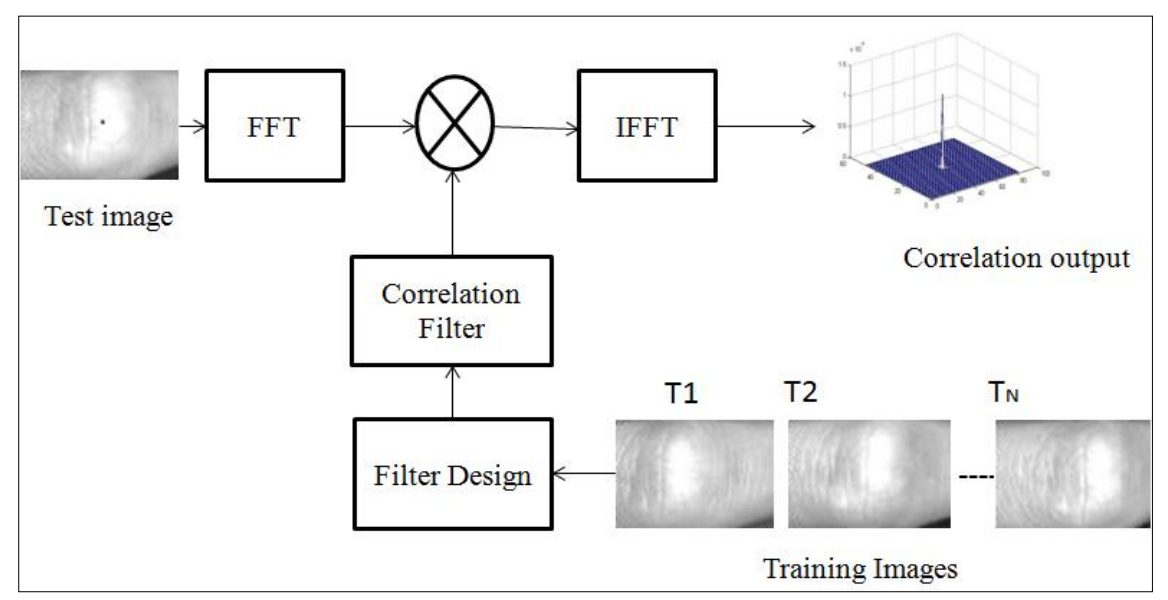

Figure 2. Schematic for the recognition process using a correlation filter 


\section{PERFORMANCE ANALYSIS}

The performance of the proposed technique was evaluated by measuring the peak-to-side lobe ratio (PSR) ${ }^{16}$. The PSR can be defined as:

$$
\mathrm{PSR}=\frac{\text { Peak-Mean }}{\text { Standard deviation }}
$$

In order to find the maximum peak value, a correlation patch of size $5 \times 5$ pixels around the peak location was selected. The maximum peak value was then calculated for this region. After that a side lobe region of size $20 \times 20$ pixels was chosen around the same peak location by excluding the patch of size $5 \times 5$ pixels. The mean and the standard deviation of this region were computed to calculate the PSR values. The PSR were used to measure the sharpness of the correlation peak. Test images were recognized as genuine if the PSR had a high value. The imposter images gave low PSR values.

For a given verification PSR threshold value, the performance can be measured by the false acceptance ratio (FAR) and false rejection ratio (FRR), defined as:

$$
\begin{gathered}
\text { FAR }=\frac{\text { Number of impostor image having PSR }>\text { threshold value }}{\text { Total number of imposter images }} \\
\text { FRR }=\frac{\text { Number of authentic images having PSR } \square \text { threshold value }}{\text { Total number of authentic images }}
\end{gathered}
$$

The equal error rate (EER) is the point where FAR and FRR are equal.

\section{RESULTS AND DISCUSSION}

A numerical experiment was carried out on a Matlab platform for the finger knuckle print verification using the correlation filter technique. The performance of the designed MACE filter for each subject was evaluated in terms of PSR and EER on a FKP image database. The numerical simulations were demonstrated on the Poly U FKP images database of Hong Kong Polytechnic University ${ }^{17}$. The FKP database consists of 165 subjects consisting of 125 males and 25 females. These FKP images were captured in two separate sessions. Each subject in the database has 48 FKP image from four fingers. This database contains 7,920 images from 660 different fingers.

In the present study, a set of 500 FKP images of 50 subjects which consist of ten FKP images of each subject were used. As explained earlier, a single MACE filter is synthesized for each subject using a set of training 
images. The cross correlation for the designed filter and the test images was obtained for 500 images in which ten images belonged to true class images and 490 images belonged to false class images. The correlation output is shown in Figs. 3 (a) and 3(b), respectively. A sharp peak was obtained for the genuine images and multiple peaks for imposter images.
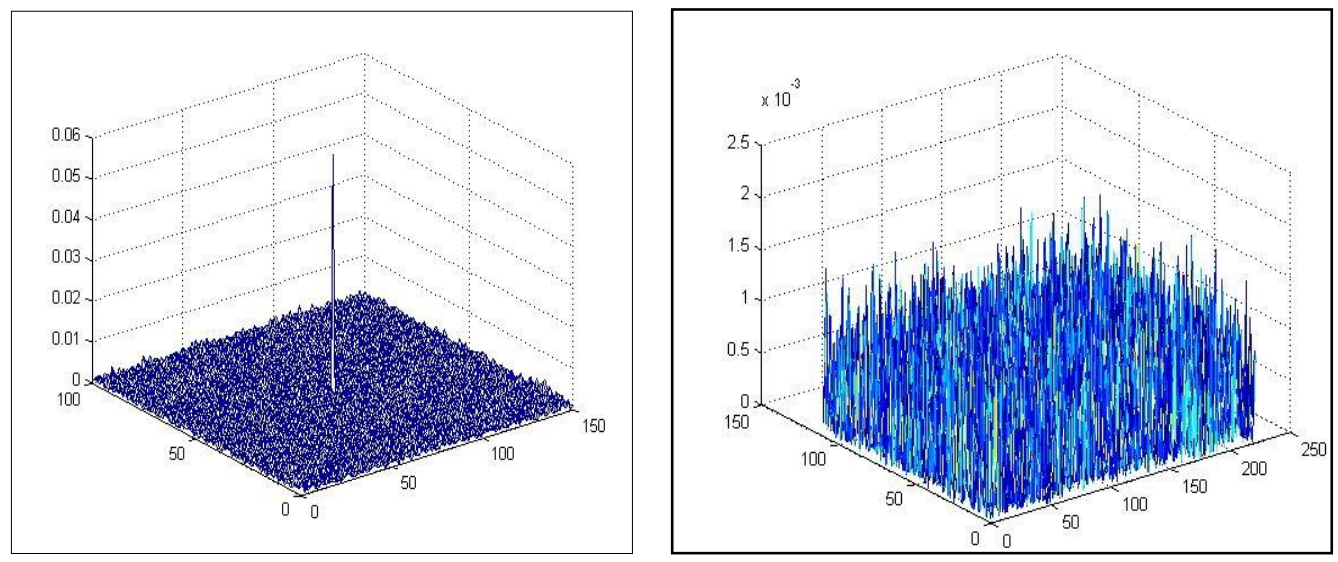

Figure 3. MACE filter output for a person: (a) Genuine, (b) Imposter

The value of PSR was computed using Eq. (12). A large value of PSR was obtained for an authentic FKP image. A value of PSR less than ten ( $<$ 10) was obtained for an imposter FKP image. For the performance evaluation, a graph was plotted between PSR and the number of images. The PSR performance of the MACE filter is shown in Figs. 4(a) and (b) for subjects 1 and 2, respectively. The PSR values of authentic images are represented by the dashed line and imposter PSRs are shown by a dotted line. It is observed from the PSR graph that for all the false class FKP images $(10 \times 49=490)$ had values of PSR of less than 10 and are shown at the bottom of the graph. The PSR values of the three training images that were used to synthesize the MACE filter are shown in the graph as the highest peak values. 


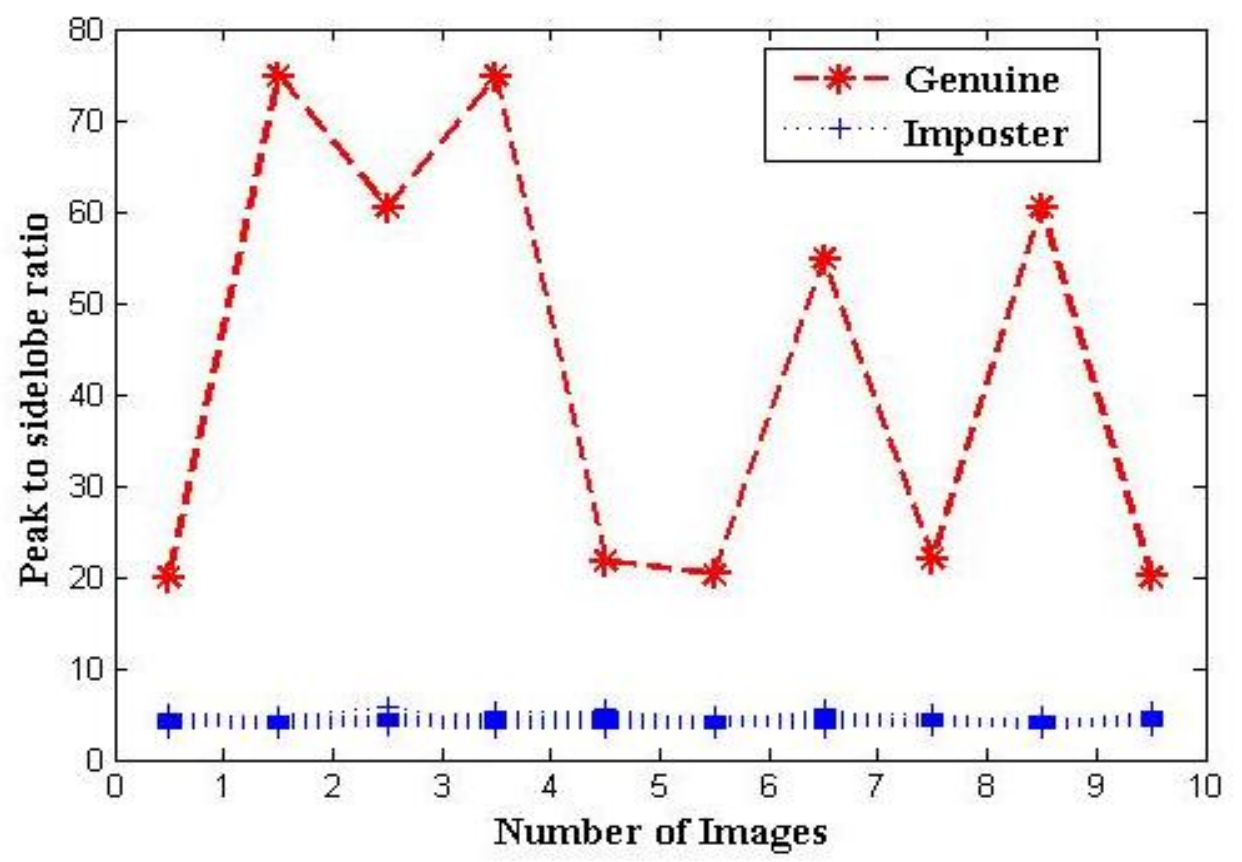

Figure 4. (a) Graph of PSR performance for subject 1

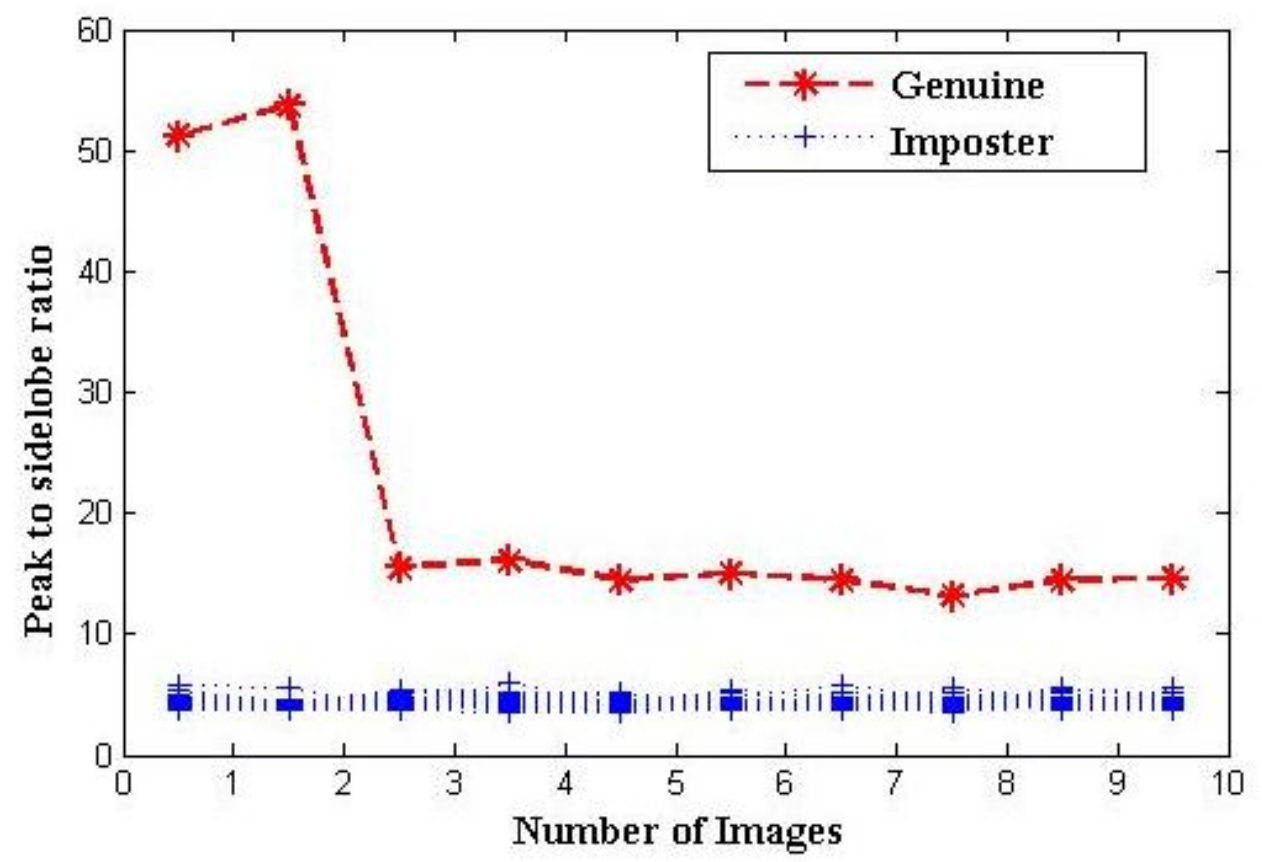

Figure 4. (b) Graph of PSR performance for subject 2 
The performance of the system was further evaluated by calculating the equal error rate. The false acceptance ratio and the false rejection ratio are determined by using the absolute difference of the PSR values. For each subject, a single PSR value was chosen as the reference PSR. The absolute difference of this reference PSR value with the genuine and imposter images was calculated. The absolute difference of PSR was then further normalized. This normalized absolute difference of the PSR values was plotted against the number of images for subjects 1 and 2 as shown in Figs. 5(a) and (b), respectively. These graphs show the distribution of the normalized absolute difference of the PSR values for a genuine and imposter population. As seen from Figs. 5(a) and (b), the normalized absolute difference of the PSR values lies between .9 to 1 for almost all imposter images, while for the genuine population this value is approximatly less then 0.9 for all the images. It can be clearly seen from Fig. 5(a) for subject 1 that the designed correlation filter shows a high discrimnation ability between a genuine and imposter population. It is observed from Fig. 5(b) for subject 2 that a small overlap area was obtained between the genuine and the imposter population. Figs. 5(a) and (b) show that the designed correlation filter performs better for subject 1 in comparison to subject 2 .

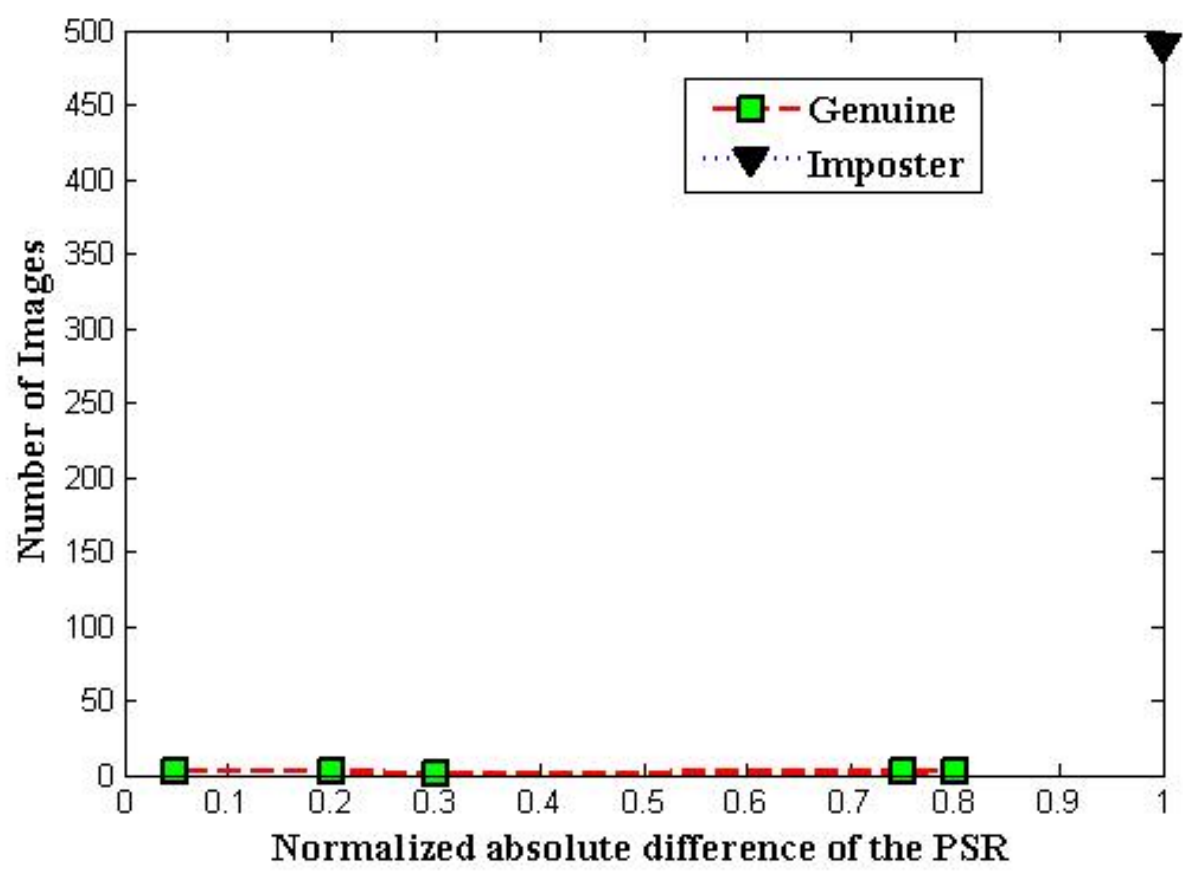

Figure 5. (a) Graph between number of images and normalized absolute difference of the PSR for subject 1 


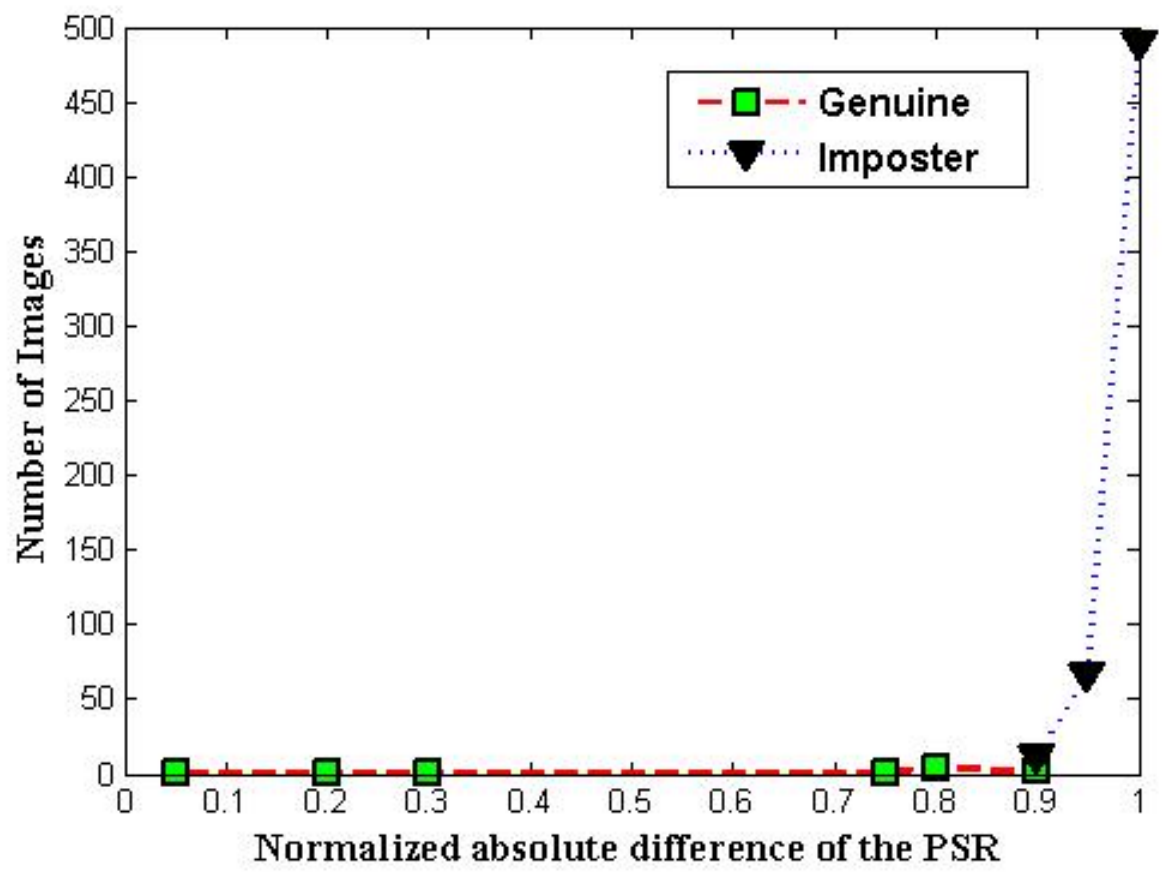

Figure 5. (b) Graph between number of images and normalized absolute difference of the PSR for subject 2

The EER values obtained for the designed MACE filter are shown in Table 1. The EER were calculated for 10 different synthesized MACE filters. As shown in Table 1, for nine subjects the designed correlation filter gave a zero EER and for subject 2 low values of EER. This indicates that the designed MACE filters for nine subjects showed high discrimination capability and for one subject a low performance.

Table 1. EER calculation for the MACE filter

\begin{tabular}{cccc}
\hline Subject & $\begin{array}{c}\text { FAR, } \\
\text { FRR }=0\end{array}$ & $\begin{array}{c}\text { FRR, } \\
\text { FAR=0 }\end{array}$ & EER \\
\hline 1 & 0 & 0 & 0 \\
2 & 2 & 12 & 1 \\
3 & 0 & 0 & 0 \\
4 & 0 & 0 & 0 \\
5 & 0 & 0 & 0 \\
6 & 0 & 0 & 0 \\
7 & 0 & 0 & 0 \\
8 & 0 & 0 & 0 \\
9 & 0 & 0 & 0 \\
10 & 0 & 0 & 0 \\
\hline
\end{tabular}


The proposed technique was compared with the results of other FKP verification based methods ${ }^{7,9}$. A comparative list of earlier results with the present proposed method are given in Table 2 .

Table 2. Comparison with earlier techniques

\begin{tabular}{ccc}
\hline Authors & Techniques & EER (\%) \\
\hline Kumar et al. ${ }^{7}$ & (PCA+ICA)*LDA*FG & 1.39 \\
Zhang et al. ${ }^{9}$ & Gabor filters & 0.26 \\
Proposed method & MACE filters & 0.1 \\
\hline
\end{tabular}

It is can be observed that the proposed method possesses a low EER and hence shows an improved recognition performance.

\section{CONCLUSION}

A MACE filter was designed to verify the efficiency of a FKP-based recognition system. The proposed correlation filtering technique was demonstrated on the FKP images of a Poly $U$ database. The performance of the MACE filter for finger knuckle print verification was evaluated in terms of PSR and EER. A graph between the PSR values and number of images was plotted and showed good discrimination ability for the FKP recognition system. The discrimination of finger knuckle print was also evaluated by plotting a graph between the number of images and the absolute difference values of PSR. Based on the obtained computational experiment results, the designed filter was able to discriminate by finger knuckle print recognition between true class samples from those that were false.

\section{REFERENCES}

[1] A.K. Jain, P. Flynn, and A. Ross, Handbook of biometrics. New York: Springer, 2008. http://dx.doi.org/10.1007/978-0-387-71041-9.

[2] A.K. Jain, A. Ross, and S. Prabhakar, An introduction to biometric recognition. IEEE Transaction on Circuits and Systems for Video Technology, 14(1), p4-20, 2004. http://dx.doi.org/10.1109/TCSVT.2003.818349.

[3] M. Savvides, B.V.K. Vijaya Kumar, and P. Khosla, Face Verification using Correlation Filters. In Ruud M. Bolle and Nalini K. Ratha (Eds.), Proceeding of Third IEEE Automatic Identification Advanced Technologies (p56-61). Tarrytown, NY: the IEEE Robotics and Automation Society in association with AIM, Inc, 2002.

[4] K. Venkataramani, and B.V.K. Vijaya Kumar, Fingerprint verification using correlation filters. Audio- and Video-Based Biometric Person 
Authentication, Lecture Notes in Computer Science, 2688, p886-894, 2003. http://dx.doi.org/10.1007/3-540-44887-X_103.

[5] B.V.K. Vijaya Kumar, C. Xie, and J. Thornton, Iris verification using correlation filters. Audio- and Video-Based Biometric Person Authentication, Lecture Notes in Computer Science, 2688, p697-705, 2003. http://dx.doi.org/10.1007/3-540-44887-X_81.

[6] D.L. Woodard, and P.J. Flynn, Finger surface as a biometric identifier. Computer Vision and Image Understanding, 100(3), p357-384, 2005. http://dx.doi.org/10.1016/j.cviu.2005.06.003.

[7] A. Kumar, and C. Ravikanth, Personal authentication using finger knuckle surface. IEEE Transaction Information Forensics and Security, 4(1), p98-109, 2009. http://dx.doi.org/10.1109/TIFS.2008.2011089.

[8] L. Zhang, L. Zhang, and D. Zhang, Finger knuckle print verification based on band-limited phase only correlation. Computer Analysis of Images and Patterns, Lecture Notes in Computer Science, 5702, p141-148, 2009. http://dx.doi.org/10.1007/978-3-642-03767-2_17.

[9] L. Zhang, L. Zhang, D. Zhang, and H. Zhu, Online finger knuckle print verification for personal authentication. Pattern Recognition, 43(7), p2560-2571, 2010. http://dx.doi.org/10.1016/j.patcog.2010.01.020.

[10] G. Ravichandran, and D. Casasent, Minimum noise and correlation energy optical correlation filter. Applied Optics, 31(11), p1823-1833, 1992. http://dx.doi.org/10.1364/AO.31.001823.

[11] A.B. VanderLugt, Signal detection by complex spatial filtering. IEEE Transaction Information Theory, 10(2), p139-145, 1964. http://dx.doi.org/10.1109/TIT.1964.1053650.

[12] C.F. Hester, and D. Casasent, Multivariant technique for multiclass pattern recognition. Applied Optics, 19(11), p1758-1761, 1980. http://dx.doi.org/10.1364/AO.19.001758.

[13] B.V.K. Vijaya Kumar, Tutorial survey of composite filter designs for optical correlators. Applied Optics, 31(23), p4773-4801, 1992. http://dx.doi.org/10.1364/AO.31.004773.

[14] A. Mahalanobis, B.V.K. Vijaya Kumar, and D. Casasent, Minimum average correlation energy filters. Applied Optics, 26(17), p3633-3630, 1987. http://dx.doi.org/10.1364/AO.26.003633.

[15] B.V.K. Vijaya Kumar, M. Savvides, K. Venkataramani, and C. Xie, Spatial frequency domain image processing for biometric recognition. In B. Mercer (Ed.), Proceedings of International Conference on Image Processing (p53-56). New York: IEEE Press, 2002. http://dx.doi.org/10.1109/ICIP.2002.1037957.

[16] A. Mahalanobis, and D. Casasent, Performance evaluation of minimum average correlation energy filters. Applied Optics, 30(5), p561-572, 1991. http://dx.doi.org/10.1364/AO.30.000561. 
[17] The Hong Kong Polytechnic university. Retrieved on December 19, 2011, from http://www.comp.polyu.edu.k/_biometrics/FKP.htm. 